Abbreviated Key Title: Sch J Med Case Rep

ISSN 2347-9507 (Print) | ISSN 2347-6559 (Online)

Journal homepage: http://saspjournals.com/sjmcr

\title{
Bilateral Peritonsillar Abscesses: A Rare Case
}

Mahiou Noureddine*, Karim Khawla, Allouch Ihssan, EL Messoudi Lina, Nitassi Sophia, Bencheikh Razika, Oujilal Abelilah, Benbouzid Anas, Essakalli Leilla

Department of Otolaryngology, Head and Neck Surgery, Ibn Sina University Hospital, Mohammed V University, Rabat, Morocco

DOI: $\underline{10.36347 / \text { sjmcr.2020.v08i07.009 }}$

| Received: 19.10.2019 | Accepted: 26.10.2019 | Published: 21.07.2020

*Corresponding author: Mahiou Noureddine

\section{Abstract}

Peritonsillary abscess (PTA) is a rare complication of acute tonsillitis. It usually presents an odynophagia, a trismus and a muffled voice. The examination reveals a purulent unilateral collection on both sides of the soft palate causing an obstruction of the upper airways; bilateral PTA is a rare event with grave consequences. Here, we describe a rare case of this pathology, presented in a public hospital and a literature review on this topic.

Keywords: Peritonsillary abscess (PTA), acute tonsillitis, odynophagia.

Copyright @ 2020: This is an open-access article distributed under the terms of the Creative Commons Attribution license which permits unrestricted use, distribution, and reproduction in any medium for non-commercial use (NonCommercial, or CC-BY-NC) provided the original author and source are credited.

\section{INTRODUCTION}

Tonsillary abscess is one of the most common cervical infections. The infection can begin with a simple tonsillitis passing through a peritonsillar cellulitis and going up to a tonsillar abscess. Unilateral involvement is common, but bilateral is rare $[1,2]$. The overall incidence of bilateral PTA would reach $4.9 \%$ [35].

\section{Case Summary}

A 9-year-old girl presented to the emergency department with a history of intermittent odynophagia, voice changes, and fever for 10 days. Prior to her emergency visit, she had been treated with amoxicillin with little improvement. She was otherwise healthy. Our examination revealed bilateral hypertrophy of the soft palate with a median uvula pushed forward (Figure1). A cervical computed tomography CT scan showed bilateral peritonsillar abscesses (Figure-2). The otolaryngologist performed a bilateral needle aspiration and examined the fluid. Subsequently, an incision was made with general anesthesia; The left side drained $15 \mathrm{cc}$ of pus, and $10 \mathrm{cc}$ of pus was evacuated from the lright peritonsillar area. The patient had a complete resolution of symptoms and was discharged and followed up with 14-day antibiotic treatment. Followup after discharge from the emergency department revealed total remission.

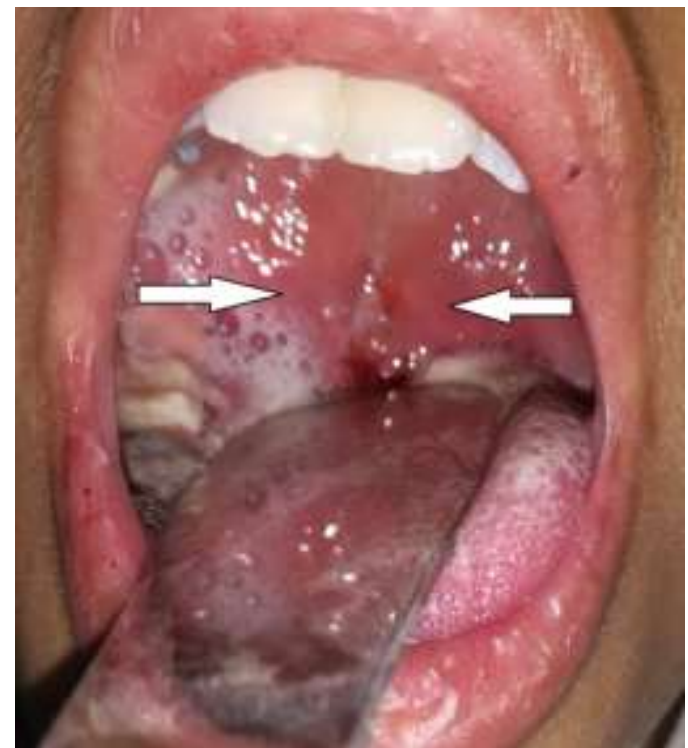

Fig-1: Bilateral hypertrophy of the soft palate (white arrow) with a median ovula pushed forward 


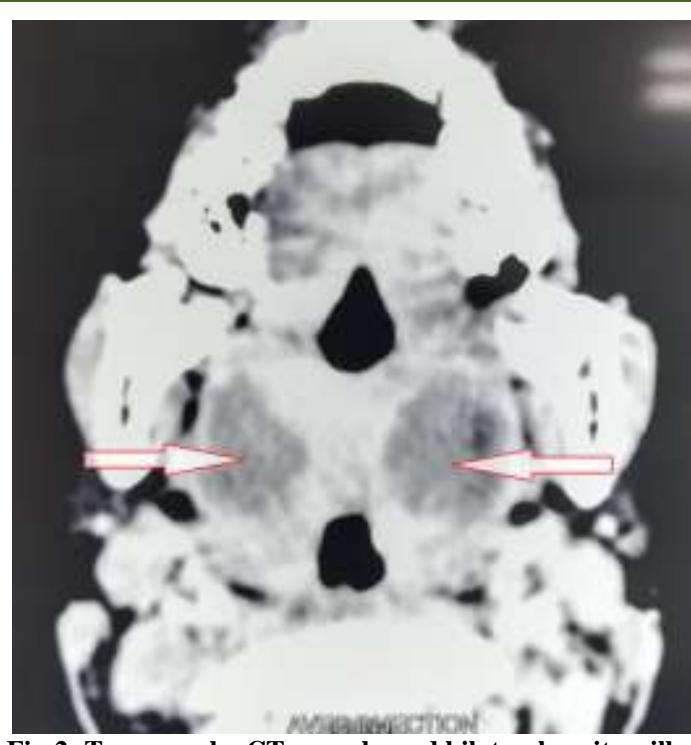

Fig-2: Tomography CT scan showed bilateral peritonsillar abcesses (white arrow)

\section{DISCUSSION}

PTA is one of the most common infections. This condition can occur in all age groups, but the high frequency in adults aged 20 to 40 years [6] in the literature, is a rare event.

This low incidence of peritonsillar abscess could be explained by early diagnosis and treatment before contralateral involvement, antibiotic treatment or underreporting.

The diagnosis should be made in all patients with infectious signs; trismus, odynophagia and having in clinical examination: advanced centralized uvula with PTA swelling [7].

Computed tomography with enhanced contrast is also useful for confirming the diagnosis of bilateral PTA and to help differentiate it from other diseases, such as acute lymphoma or tonsillitis, and evaluate extensions in deep neck spaces and even sometimes guide drainage in some difficult cases [8].

The treatment of a PTA is controversial. The treatment consists of administering antibiotherapy associated with either drainage of the abscess using a suction needle or an incision and rarely tonsillectomy. There is no agreement on the optimal technique for the initial drainage of a peritonsillar abscess [9].

The first risk of bilateral PTA is obstruction of the upper respiratory tract, unlike unilateral lesions. In addition, the risk of extension to the deep spaces of the neck is high. Consequences of these risks; Rapid diagnosis followed by appropriate treatment is essential. According to a study by Klug TE, smoking is a risk factor in favor of abstaining which increases the risk to $150 \%$ compared to non-smokers; This may explain why the pathology is more common in men than from women. A history of recurrent tonsillitis would also be one of the risk factors; It has been reported that 10 to $79 \%$ of patients with PTA had a history of tonsillitis [9].

Early administration of antibiotics can prevent the deterioration and progression of acute tonsillitis to abscess formation.14 Tonsillectomy is performed in cases of recurrent episodes of peritonsillar abscess or recurrent tonsillitis. The family doctors are a very important role in decreasing frequency of this sort of infection, referred tonsillitis to specialists for a tonsillectomy. Smoking cessation is also thought to reduce the incidence because there is a strong association between themes [9].

\section{CONCLUSION}

Awareness of this rare presentation of bilateral PTA; It should be remembered that a precocious diagnosis and adequate treatment may limit the risk of this disease.

\section{REFERENCES}

1. Fasano CJ, Chudnofsky C, Vanderbeek P. Bilateral peritonsillar abscesses: not your usual sore throat. The Journal of emergency medicine. 2005 Jul 1;29(1):45-7.

2. Loh J, Sakina SM, Husain S. Bilateral peritonsillar abscess: A rare variant. Rawal Medical Journal. 2009 Jul;34(2):236-7.

3. Dalton RE, Abedi E, Sismanis A. Bilateral peritonsillar abscesses and quinsy tonsillectomy. Journal of the National Medical Association. 1985 Oct;77(10):807-812.

4. Simons JP, Branstetter IV BF, Mandell DL. Bilateral peritonsillar abscesses: case report and literature review. American journal of otolaryngology. 2006 Nov 1;27(6):443-5.

5. Kanesada K, Mogi G. Bilateral peritonsillar abscesses. Auris Nasus Larynx. 1981 Jan $1 ; 8(1): 35-9$.

6. Galioto NJ. Peritonsillar Abscess. Am Fam Physician. 2008 Jan; 77(2):199-202.

7. Loh J, Sakina SM, Husain S. Bilateral peritonsillar abscess: A rare variant. Rawal Medical Journal. $2009 \mathrm{Jul} ; 34(2): 236-7$

8. Boon C, Mohamad WW, Mohamad I. Bilateral peritonsillar abscess: A rare emergency. Malaysian family physician: the official journal of the Academy of Family Physicians of Malaysia. 2018;13(1):41-44.

9. Klug TE. Peritonsillar Abscess: Clinical Aspects of Microbiology, Risk Factors, and the Association with Parapharyngeal Abscess. Dan Med Journal. 2017 Mar; 64(3):B5333. 